\title{
A Non-Classical Optimal Control Problem
}

\section{Wan Noor Afifah Wan Ahmad, Suliadi Sufahani*, Mohd Saifullah Rusiman, Maselan Ali}

Department of Mathematics and Statistics, Faculty of Applied Sciences and Technology, Universiti Tun Hussein Onn Malaysia, Pagoh Educational Hub, 84600 Pagoh, Johor, Malaysia.

Received 6 February 2018; accepted 30 May 2018; available online 27 June 2018

DOI: https://10.30880/jst.2018.10.01.005

\begin{abstract}
We consider another non-classical of optimal control problem that is spurred by some current research on the nonlinear income issue in the field of financial matters. This class of issue can be set up as a maximizing issue in the area of Optimal Control. In any case, the state value at the final fixed time, $y(T)$, is priori unknown and the integrand is an element of the unknown $y(T)$. This is a non-classical optimal control problem. In this paper we apply the new costate value conditions $p(T)$ in the definition of the optimal control problem. We solve some examples in this issue using the numerical shooting method to illuminate the subsequent Two Point Boundary Value Problem (TPBVP) and join the free $y(T)$ as an additionally unknown. Basically similar outcomes are obtained through the nonlinear programming (NP) discrete-time results.
\end{abstract}

Keyword: Mathematics; Calculus of Variation; Optimal Control Problem; Nonlinear Programming; Shooting Method

\section{Introduction}

Calculus of Variations $(\mathrm{CoV})$ gives the mathematical theory to take care of extremizing functional issues for which a given functional has a stationary value either minimum or maximum [8]. Optimal control is an expansion of $\mathrm{CoV}$ and it is a mathematical optimization method for determining optimal control strategies. A couple of standard cases that mirror the utilization of optimal control are the medication bust technique, optimal generation, optimal control in discrete mechanics, strategy plan and the royalty installment issue $[3,4,7]$. Consider the framework in the time area displayed by the differential equation

$$
y^{\prime}(t)=u(t), y(0) \text { known }
$$

with the unknown endpoint of state value $y(T)$ at time $t=T$. We wish to decide the control function $u(t)$ for $t \in[0, T]$ that maximizes

$$
J[u(\cdot)]=\int_{0}^{T} f(t, y(t), u(t), y(T)) d t
$$

Note that the integrand relies on the priori unknown final value, $y(T)$. This paper is sorted out as follows. In Section 2, we build up the necessary conditions for the extremizing solution. Then in Section 3, we consider a simple illustrative example. We finish the last section with conclusions.

\section{The Non-Classical Optimal Control Problem}

We start by building up the necessary conditions for the extremizing solution. Suppose $J$ be a functional of the form

$$
J[y(\cdot)]=\int_{a}^{T} f\left(t, y(t), y^{\prime}(t), y(T)\right) d t
$$

where $T>a$. We consider the issue of deciding the functions $y(\cdot) \in C^{1}$ with the end goal that $J[\cdot]$ has an extremum. An underlying condition $y(a)=\alpha$ is forced on $y(\cdot)$, however $y(T)$ is unknown.

Assume that $J[\cdot]$ has an extremum at 9/(.). We can continue as Lagrange did [2], by considering the estimation of $J$ at a close- 
by function $y=f / \theta+\varepsilon h$, where $\varepsilon$ is a small parameter, $h(\cdot) \in C^{1}$ and $h(a)=0$. Since $y(T)$ is unknown, we do not expect $h(\cdot)$ to vanish at $T$. Let

$$
\begin{aligned}
\phi(\varepsilon) & =J[(f / t+\varepsilon h)(\cdot)] \\
& =\int_{a}^{T} f\left(\begin{array}{l}
t, f(\phi t)+\varepsilon h(t), f y(t)+\varepsilon h^{\prime}(t), \\
f l(T)+\varepsilon h(T)
\end{array}\right) d t
\end{aligned}
$$

A fundamental condition for $\mathscr{y}(\cdot)$ to be an extremizer is given by

$$
\begin{aligned}
& \left.\phi^{\prime}(\varepsilon)\right|_{\varepsilon=0}=0 \\
& \Leftrightarrow \int_{a}^{T}\left[\begin{array}{l}
f_{y}(\mathrm{~L}) h(t)+f_{y^{\prime}}(\mathrm{L}) h^{\prime}(t)+ \\
f_{z}(\mathrm{~L}) h(T)
\end{array}\right] d t=0
\end{aligned}
$$

where $(\mathrm{L})=(t, \rho /(t), g / t(t), g /(T))$.

Integration by parts gives

$$
\begin{aligned}
& \int_{a}^{T} f_{y^{\prime}}(\mathrm{L}) h^{\prime}(t) d t \\
& \left.=f_{y^{\prime}}(\mathrm{L}) h(t)\right]_{a}^{T}-\int_{a}^{T} \frac{d}{d t}\left(f_{y^{\prime}}(\mathrm{L})\right) h(t) d t
\end{aligned}
$$

Since $h(a)=0$, the necessary condition (4) can be then composed as

$$
0=\int_{a}^{T}\left\{\begin{array}{l}
{\left[\begin{array}{l}
\left.f_{y}(\mathrm{~L})-\frac{d}{d t} f_{y^{\prime}}(\mathrm{L})\right] h(t)+ \\
{\left[\frac{f_{y^{\prime}}(T, \phi(T), \phi y(T), \phi(t))}{T-a}+\right.} \\
f_{z}(\mathrm{~L})
\end{array}\right] h(T)}
\end{array}\right\} d t
$$

for all $h(\cdot) \in C^{1}$ with the end goal that $h(a)=0$. Specifically, condition (8) holds for the subclass of function $h(\cdot) \in C^{1}$ that do vanish at $h(T)$.Hence, the classical arguments apply, and in this way

$$
f_{y}(\mathrm{~L})-\frac{d}{d t} f_{y^{\prime}}(\mathrm{L})=0
$$

Condition (7) must be fulfilled for all $h(\cdot) \in C^{1}$ with $h(a)=0$, which incorporates functions $h(\cdot)$ that do not vanish at $T$. Thus, conditions (7) and (8) infer that

$$
\begin{aligned}
& \int_{a}^{T}\left[\frac{f_{y^{\prime}}(T, g /(T), \varphi g(T), g /(T))}{T-a}+f_{z}(\mathrm{~L})\right] h(T) d t=0 \\
& \Leftrightarrow h(T)\left(f_{y^{\prime}}(T, g /(T), g / c(T))+\int_{a}^{T} f_{z}(\mathrm{~L}) d t\right)=0
\end{aligned}
$$

That is,

$$
f_{y^{\prime}}(T, \varphi /(T), \varphi l(T), \oint /(T))+\int_{a}^{T} f_{z}(\mathrm{~L}) d t=0
$$

Note that in the function $f$ does not depend on $y(T)$ in the classical setting, which is, $f_{z}=0$. All things considered (10) lessens to the outstanding normal boundary condition $f_{y^{\prime}}(T, f /(T), f y(T))=0$ (or, from a Hamiltonian optimal control point of view, $p(T)=0)$.We have quite recently demonstrated the accompanying outcome:

Theorem 2.1: Let $a$ and $T$ be given real numbers, $a<T$. If $f /(\cdot)$ is a solution of the problem 


$$
\begin{aligned}
& J[y(\cdot)]=\int_{a}^{T} f\left(t, y(t), y^{\prime}(t)\right. \\
& y(a)=\alpha, \quad(y(T) \text { free }) \\
& y(\cdot) \in C^{1},
\end{aligned}
$$

then

$$
\begin{aligned}
& \frac{d}{d t} f_{y^{\prime}}(t, \rho /(t), g / d(t), \rho /(T)) \\
& =f_{y}(t, g /(t), g /(t), g(T))
\end{aligned}
$$

for all $t \in[a, T]$. Moreover,

$$
\begin{aligned}
& f_{y^{\prime}}(T, \oint /(T), \oint f(T), \oint /(T)) \\
& =-\int_{a}^{T} f_{z}(t, g /(t), g / d(t), g /(T)) d t
\end{aligned}
$$

From an optimal control point of view one has

$$
p(T)=f_{y^{\prime}}(T, \rho /(T), \rho / d(T), g /(T))
$$

where $p(t)$ is the Hamiltonian multiplier. Theorem 2.1 states that the standard necessary optimality conditions (the Euler-Lagrange equation [2] or the Pontryagin maximum principle [5] hold for issue (11) by substituting the classical transversality condition $p(T)=0$ with

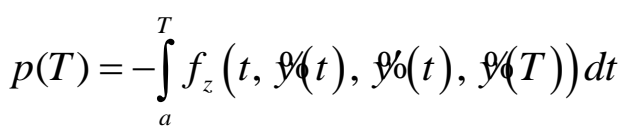

\section{Numerical Example}

Consider the Ordinary Differential Equation system below

$$
y^{\prime}(t)=u(t), y(0)=0 .
$$

We wish to maximize

$$
J[u(\cdot)]=\int_{0}^{T} f(t, y(t), u(t), z) d t
$$

where

$$
f(t, y, u, z)=a \sqrt{u}-\left(\frac{1}{2}+z \sin \left(\frac{\pi t}{10}\right)\right) u
$$

is a continuous function. The initial known state is $y(0)=0$ and final state value $z=y(T)$ is unknown. In this paper, we set $T=50$. The Hamiltonian is $H(t, y, u, p)=f+p \cdot u$ and

$$
\left\{\begin{array}{c}
y^{\prime}(t)=H_{p}(t, y(t), u(t), p(t)) \\
p^{\prime}(t)=-H_{y}(t, y(t), u(t), p(t)) .
\end{array}\right.
$$

Function $f$ does not rely upon $y$ and for an ideal (maximum in this illustration) the costate fulfills

$$
p^{\prime}=-H_{y} \Leftrightarrow p^{\prime}=0
$$

The stationary condition is

$$
H_{u}=0
$$

and this yields

$$
u(t)=\frac{1}{4}\left(z \sin \left(\frac{\pi t}{10}\right)-p(t)\right)^{2}
$$

From (15)

$$
p(T)=-\int_{0}^{50} f_{z}(t, 9(\phi t), \varphi d(t), \oint(1 T)) d t
$$

Holds

$$
p(T)=\int_{0}^{50} \sin \left(\frac{\pi t}{10}\right) u(t) d t
$$

\section{Results}

Let us consider the necessary condition that should be fulfilled. For the system of Ordinary Differential Equations (16) and (20) with control (22), the known zero initial condition 
$y(0)$ and a guessed initial value $p(0)$, we have to guarantee that the normal boundary condition (24) is fulfilled.

The two point boundary value problem need to be solved. The value of $z$ that has been used in (22) also need to iterate so that the $z$ value obtained will be the same as $y(t)$ at $t=T$. When one has gotten convergence in regards to the values $y(T)$ utilized as a part of (22) and $p(T)$ (24), at that point the necessary condition is fulfilled and we ought to have the optimal solution.

Utilize the Newton shooting technique with two guessed value $v_{1}$ and $v_{2}$ [1]. We want $v_{1}=p(0)$ and $v_{2}=p(T)$ as determined by condition (24). At the point when the program acquires the outcome with these two conditions holding to a high level of accuracy, the necessary conditions hold and we ought to have the optimal solution. We have tackled the shooting technique issue utilizing $\mathrm{C}++$ and the profoundly precise Numerical Formulas library routines [6].

Integrate the system of Ordinary Differential Equations (16) and (20), and

$$
\begin{aligned}
& g_{p}^{\prime}=\sin \left(\frac{\pi t}{10}\right) u(t) \\
& J^{\prime}=g
\end{aligned}
$$

The outcomes are $y(T)=0.900000$, $p(T)=0.015431, g_{p}(T)=-3.275093$ and $J(T)=6.25257$

\section{Conclusions}

In this paper we have demonstrated to solve a nonstandard optimal control issue. We have introduced the fundamental conditions and computational techniques so as to acquire optimal solutions. A shooting method together with an expanding approach was utilized to acquire an exceedingly precise solution and compared with a discrete-time nonlinear programming solution. Our systems can be connected to the real problem rather more entangled financial matters issue where the Lagrangian integrand is piecewise constant in many stages and relies on the $y(T)$ which is priori unknown.

\section{Acknowledgements}

The authors would like to thank the responsible community of Faculty of Applied Sciences and Technology, UTHM for giving a useful opportunity to publish paper. We are additionally grateful to anonymous referees and journal editors for their careful reading the paper and insightful comments and remarks that assist us in enhancement of this paper.

\section{References}

[1] Betts, J. T. (2001). Practical Methods for Optimal Control Using Nonlinear Programming. Advances in Design and Control, Society for Industrial and Applied Mathematics (SIAM), Philadelphia, PA.

[2] Gelfand, I. M. \& Fomin, S. V. (1963). Calculus of Variations. Revised English Edition Translated and Edited by Richard A. Silverman, Prentice Hall, Englewood Cliffs, N. J.

[3] Leonard, D. \& Long, N. V. (1992). Optimal Control Theory and Static Optimization in Economics. Cambridge University Press, Cambridge.

[4] Pedro, A. F. C., Torres, D. F. M. \& Zinober, A. S. I. (2010). "A Non Classical Class of Variational Problems with Application in Economics" in International Journal of Mathematical Modeling and Numerical Optimization, Vol. 1. No. 3. pp. 227-236.

[5] Pontryagin, L. S., Boltyanskii, V. G., Gamkrelidze, R. V. \& Mishchenko, E. F. (1986). Selected Works. The Mathematical Theory of Optimal Processes. Translated from the Russian by K. N. Trirogoff and Translation Edited by L. W. Neustadt, Reprint of the 1962 English Translation, Gordon \& Breach, New York.

[6] Press, W. H., Teukolsky, S. A., Vetterling, W. T. \& Flannery, B. P. (2007). Numerical Recipes - The Art of Scientific Computing. Cambridge University Press, Cambridge.

[7] Sethi, S. P. \& Thompson, G. L. (2000). Optimal Control Theory - Applications on 
Journal of Science and Technology, Vol. 10 No. 1 (2018) p. 28-32

Management Science and Economics. Kluwer Academic Publishers, Boston, MA.

[8] Pinch, E. R. (1993). Optimal Control and the Calculus of Variations. Oxford University Press, Oxford. 